\title{
Distributed Space-Time Cooperative Schemes for Underwater Acoustic Communications
}

\author{
Madhavan Vajapeyam, Member, IEEE, Satish Vedantam, Urbashi Mitra, Fellow, IEEE, \\ James C. Preisig, Member, IEEE, and Milica Stojanovic, Senior Member, IEEE
}

\begin{abstract}
In resource limited, large scale underwater sensor networks, cooperative communication over multiple hops offers opportunities to save power. Intermediate nodes between source and destination act as cooperative relays. Herein, protocols coupled with space-time block code (STBC) strategies are proposed and analyzed for distributed cooperative communication. Amplify-and-forward-type protocols are considered, in which intermediate relays do not attempt to decode the information. The Alamouti-based cooperative scheme proposed by Hua et al. (2003) for flat-fading channels is generalized to work in the presence of multipath, thus addressing a main characteristic of underwater acoustic channels. A time-reversal distributed space-time block code (TR-DSTBC) is proposed, which extends the dual-antenna TR-STBC (time-reversal space-time block code) approach from Lindskog and Paulraj (2000) to a cooperative communication scenario for signaling in multipath. It is first shown that, just as in the dual-antenna STBC case, TR along with the orthogonality of the DSTBC essentially allows for decoupling of the vector intersymbol interference (ISI) detection problem into separate scalar problems, and thus yields strong performance (compared with single-hop communication) and with substantially reduced complexity over nonorthogonal schemes. Furthermore, a performance analysis of the proposed scheme is carried out to provide insight on the performance gains, which are further confirmed via numerical results based on computer simulations and field data experiments.
\end{abstract}

Index Terms-Cooperative diversity methods, multiple-inputmultiple-output (MIMO) fading channels, underwater sensor networks.

\section{INTRODUCTION}

$\mathbf{U}$ NDERWATER sensor networks form an emerging technology paradigm that promises to enable or enhance several key applications in oceanic research, such as data collection, pollution monitoring, tactical surveillance, and disaster prevention [1], [2].

Manuscript received September 10, 2006; revised February 07, 2008; accepted August 15, 2008. Current version published February 06, 2009. This work was supported in part by one or more of the following Grants: ONR N-000140410273, NSF ITR CCF-0313392, and NSF OCE 0520324.

Associate Editor: R. Spindel.

M. Vajapeyam is with Qualcomm Inc., San Diego, CA 92121 USA (e-mail: msvajape@qualcomm.com).

S. Vedantam and U. Mitra are with the Communication Sciences Institute, University of Southern California, Los Angeles, CA 90089 USA and also with the Department of Electrical Engineering-Systems, Los Angeles, CA 900892565 USA (e-mail: vedantam@usc.edu; ubli@usc.edu).

J. Preisig is with the Department of Applied Ocean Physics and Engineering, Woods Hole Oceanographic Institution, Woods Hole, MA 02543 USA (e-mail: jpreisig@whoi.edu).

M. Stojanovic is with the Electrical and Computer Engineering Department, Northeastern University, Boston, MA 02115 USA (e-mail:millitsa@mit.edu).

Digital Object Identifier 10.1109/JOE.2008.2005338
Exploiting sensor cooperation for terrestrial communications has attracted considerable recent attention to increase reliability, coverage, throughput, and capacity (see [3] and references therein). A common feature among many of these networks is their multihop nature: communication is performed between a source and destination via intermediate terminals. This method provides several advantages over single-hop schemes [3]: 1) combatting the severe signal attenuation over long distances, and therefore, saving transmission power; 2) providing signal paths between terminals that do not have a direct line of sight between them; and 3) providing multiple communication links for applications with a high data rate requirement, which cannot be satisfied via a single link.

Multihop networks can also provide additional gains through cooperation between terminals. Recent information theoretic results show that cooperation can increase the overall capacity of these networks by taking advantage of their inherent increase in spatial diversity [4]-[6]. A natural way to exploit this diversity is via distributed space-time block coding (DSTBC) originally proposed in [6]. The goal of a DSTBC-based protocol is to allow the cooperating terminals to act, from the destination point of view, as a multiantenna array employing a well-designed space-time block code (STBC) [7]. Several DSTBC schemes have been recently proposed [6], [8]-[12].

The idea that DSTBC schemes could be applied to underwater networks suggests itself naturally. The underwater acoustic channel, however, poses additional difficulties to the design of such communication protocols. The major challenges posed by underwater channels are as follows [1]: severe range-dependent attenuation, extensive multipath propagation, and highly variable propagation delays (due to slow sound propagation).

In this paper, we consider the problem of underwater communication between a single source and a destination terminal. Data is relayed in a multihop fashion, through intermediate sensor nodes placed between the source and the destination. Communication protocols based on DSTBC are considered. The time-reversal STBC (TR-STBC) approach proposed in [13] for dual colocated antennas and signaling in multipath is extended to a distributed communication scenario. The key feature of this approach is to allow remote terminals not directly wired to the source to relay information to the destination, hence acting as virtual antennas. We show that, as in the STBC case [13], [14], TR along with the orthogonality of the DSTBC essentially allows for decoupling of a vector intersymbol interference (ISI) detection problem into separate scalar problems, without loss of optimality (neglecting "border effects" [14]) and, therefore, offers excellent performance with significant complexity reduction over nonorthogonal schemes. 


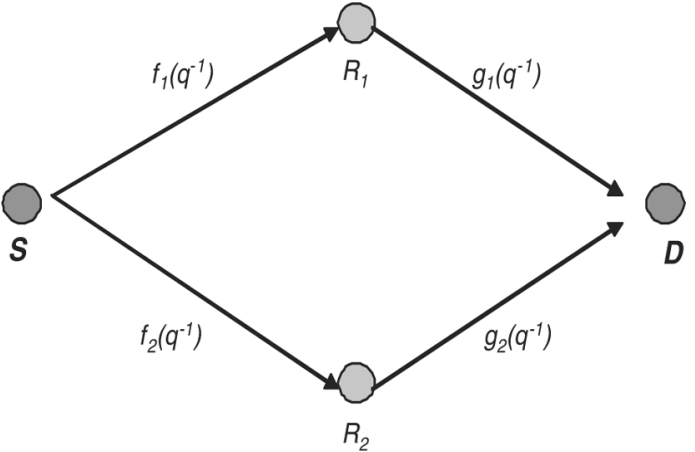

Fig. 1. Two-hop cooperative network with two relays.

This paper is organized as follows. Section II introduces the signal model and the TR-DSTBC scheme for two relays. Section III presents a diversity analysis of the proposed scheme employing a decision-feedback equalizer (DFE) at the receiver, under standard simplifying assumptions. The robustness of the scheme to asynchronous relay operation is shown in Section IV. A generalization of the scheme to more than two relays is presented in Section V, based on the rate 1/2 orthogonal STBC. The ray-based underwater channel model used in our computer simulations, which is essentially based on [15] and [16], is presented in Section VI. Numerical simulations and field test experimental results, which confirm the potential gains of the proposed approach, are presented in Section VII, which also addresses some practical issues such as imperfect channel state information (CSI) at the receiver and the presence of channel time variations. Section VIII presents the conclusions.

Throughout this paper, scalar quantities are denoted with lower or upper case normal font, vectors are denoted with bold-faced lower case fonts, and matrices are denoted with bold-faced upper case fonts. The symbol "*" denotes complex conjugate and the superscript " $H$ " denotes Hermitian (complex conjugate transpose). The distribution of a complex Gaussian random variable with mean $\mu$ and variance $\sigma^{2}$ will be denoted by $\mathcal{C N}\left(\mu, \sigma^{2}\right)$.

\section{SIGNAL MODEL}

We first consider the discrete-time signal model for a scenario with a single source terminal $(S)$ communicating to a destination terminal $(D)$ via a stage of two wireless relays as depicted in Fig. 1. Because the channels between the multiple links contain ISI, we will employ a discrete-time model. To clarify the notation, for a generic input $u(t)$ and channel filter $a\left(q^{-1}\right)$-with $q^{-1}$ denoting the delay operator-the output $v(t)$ is given by

$$
\begin{aligned}
v(t) & =a\left(q^{-1}\right) u(t)=\left(\sum_{i=0}^{L_{c}} a_{i} q^{-i}\right) u(t) \\
& =\sum_{i=0}^{L_{c}} a_{i} u(t-i), \quad t=1, \ldots, N
\end{aligned}
$$

where $L_{c}+1$ is the number of channel taps and $N$ is the block size. It is useful to note that

$$
\begin{aligned}
& v(N-t+1)=a\left(q^{-1}\right) u(N-t+1) \\
&=\left(\sum_{i=0}^{L_{a}} a_{i} q^{-i}\right) u(N-t+1), \\
& t=1, \ldots, N .
\end{aligned}
$$

Denoting the time-reversed input and output by $\bar{u}(t) \triangleq$ $u(N-t+1)$ and $\bar{v}(t) \triangleq v(N-t+1)$, respectively, we have from (2)

$$
\begin{aligned}
\bar{v}(t) & =v(N-t+1)=a\left(q^{-1}\right) u(N-t+1) \\
& =a(q) \bar{u}(t) .
\end{aligned}
$$

Let $h_{i}\left(q^{-1}\right)$ and $g_{i}\left(q^{-1}\right)$ denote the $S-R_{i}$ and $R_{i}-D$ channels, respectively. Throughout this work, we will assume that all channels are independent, with taps that are independently fading and quasi-static (time invariant for a duration $2 N$ plus any required guard intervals, as explained next).

The source divides its transmission symbol stream $s(t)$ into two blocks $s_{1}(t)$ and $s_{2}(t)$, each of length $N$ and transmits them separated by a guard band to avoid interblock interference. For the same reason, a preamble and a tail are inserted at the beginning and at the end of $s(t)$, respectively [14]. The received signal at $R_{i}$ at sampling times $t$ corresponding to the first transmission block is

$$
r_{i, 1}(t)=\sqrt{E_{s}} h_{i}\left(q^{-1}\right) s_{1}(t)+w_{i, 1}(t), \quad t=1, \ldots, N
$$

and similarly for the second transmission block

$$
r_{i, 2}(t)=\sqrt{E_{s}} h_{i}\left(q^{-1}\right) s_{2}(t)+w_{i, 2}(t), \quad t=1, \ldots, N .
$$

Thus, $r_{i, k}(t)$ is the received signal at time $t$, at relay $i$ and block $k$, where $k=1,2$. The signal $s_{k}(t)$ is the $t$ th source transmitted symbol of block $k$ and is taken from a phase-shift keying (PSK) or quadratic-amplitude modulation (QAM) symbol constellation. The additive white Gaussian noise (AWGN) sequence $w_{i, k}(t)$ has unit variance. Furthermore, $w_{i, 1}(t)$ and $w_{i, 2}(t)$ are assumed to be independent. The energy per transmit symbol is denoted by $E_{s}$.

We assume, due to complexity and power limitations, that the relays can only perform amplify-and-forward-type operations on their received signals (amplification, complex conjugation, or time shift). No channel estimation or symbol detection is performed. In the case of flat fading, the work by Hua [8] has shown how an Alamouti-type [17] processing can be employed by the relays to achieve diversity gains. We now describe its extension to multipath channels, following an approach similar to [13] and [14]. 
Both $R_{1}$ and $R_{2}$ transmit two blocks. Let $u_{i, k}(t)$ denote the signal transmitted by $R_{i}$ over block $k$ and time $t$. In the first block, $R_{1}$ and $R_{2}$ transmit, respectively, the following signals:

$$
\begin{aligned}
& u_{1,1}(t)=\sqrt{\frac{E_{r}}{K_{1}}} r_{1,1}(t) \\
& u_{2,1}(t)=\sqrt{\frac{E_{r}}{K_{2}}} \bar{r}_{2,2}^{*}(t)
\end{aligned}
$$

where $K_{i}, i=1,2$ is a normalizing factor applied to the received signal of relay $i$, to make it a unit power, and $E_{r}$ is the transmit energy per symbol for each relay.

In the second block, the transmitted signals are

$$
\begin{aligned}
& u_{1,2}(t)=\sqrt{\frac{E_{r}}{K_{1}}} r_{1,2}(t) \\
& u_{2,2}(t)=-\sqrt{\frac{E_{r}}{K_{2}}} r_{2,1}^{*}(t) .
\end{aligned}
$$

We note that $R_{2}$ conjugates and time-reverses both of its received blocks and transmits them in inverse order after amplification. This is different from the approach in [13] where two colocated antennas perform conjugation and time reversal over the second block. Although both approaches are equivalent for the STBC scenario, the approach in [13] cannot be used with relays because this would make the overall $S-D$ channel matrix nonorthogonal. ${ }^{1}$ As will be shown next, orthogonality is the key property that will allow us to decouple the problem of joint detection of $s_{1}(t)$ and $s_{2}(t)$ without loss in optimality and significant reduction in receiver complexity.

The received signal at the destination for block $k(k=1,2)$ is, therefore, given by

$$
y_{k}(t)=g_{1}\left(q^{-1}\right) u_{1, k}(t)+g_{2}\left(q^{-1}\right) u_{2, k}(t)+n_{k}(t) .
$$

Substituting the expressions for $u_{i, k}(t)$ from above, we have

$$
\begin{aligned}
y_{1}(t)= & \sqrt{\frac{E_{s} E_{r}}{K_{1}}} g_{1}\left(q^{-1}\right) h_{1}\left(q^{-1}\right) s_{1}(t) \\
& +\sqrt{\frac{E_{s} E_{r}}{K_{2}}} g_{2}\left(q^{-1}\right) h_{2}^{*}(q) \bar{s}_{2}^{*}(t)+n_{1}^{\prime}(t) \\
y_{2}(t)= & \sqrt{\frac{E_{s} E_{r}}{K_{1}}} g_{1}\left(q^{-1}\right) h_{1}\left(q^{-1}\right) s_{2}(t) \\
& -\sqrt{\frac{E_{s} E_{r}}{K_{2}}} g_{2}\left(q^{-1}\right) h_{2}^{*}(q) \bar{s}_{1}^{*}(t)+n_{2}^{\prime}(t)
\end{aligned}
$$

where

$$
\begin{aligned}
n_{1}^{\prime}(t)= & n_{1}(t)+\sqrt{\frac{E_{r}}{K_{1}}} g_{1}\left(q^{-1}\right) w_{1,1}(t) \\
& +\sqrt{\frac{E_{r}}{K_{2}}} g_{2}\left(q^{-1}\right) \bar{w}_{2,1}^{*}(t) \\
n_{2}^{\prime}(t)= & n_{2}(t)+\sqrt{\frac{E_{r}}{K_{1}}} g_{1}\left(q^{-1}\right) w_{1,2}(t) \\
& -\sqrt{\frac{E_{r}}{K_{2}}} g_{2}\left(q^{-1}\right) \bar{w}_{2,2}^{*}(t)
\end{aligned}
$$

and $n_{k}(t)$ is AWGN with unit variance. It follows, therefore, that the power spectral density (PSD) of $n_{i}^{\prime}(t)$, denoted by $X\left(q^{-1}, q\right)$, is given by

$$
X\left(q^{-1}, q\right)=1+\frac{E_{r}}{K_{1}} g_{1}\left(q^{-1}\right) g_{1}^{*}(q)+\frac{E_{r}}{K_{2}} g_{2}\left(q^{-1}\right) g_{2}^{*}(q)
$$

where we keep the PSD notation in the $q$ domain instead of switching to the more conventional domain $z$, for notational simplicity.

By computing the time reversal and conjugation of $y_{2}(t)$, we obtain

$$
\begin{aligned}
\bar{y}_{2}^{*}(t)=\sqrt{\frac{E_{s} E_{r}}{K_{1}}} & g_{1}^{*}(q) h_{1}^{*}(q) \bar{s}_{2}^{*}(t) \\
& -\sqrt{\frac{E_{s} E_{r}}{K_{2}}} g_{2}^{*}(q) h_{2}\left(q^{-1}\right) s_{1}(t)+\tilde{n}_{2}(t)
\end{aligned}
$$

where $\tilde{n}_{2}^{*}=\bar{n}_{2}^{\prime}$. Defining $\mathbf{y}(t)=\left[y_{1}(t) \bar{y}_{2}^{*}(t)\right]^{T}$, we have

$$
\mathbf{y}(t)=\mathbf{H}\left(q^{-1}, q\right)\left[\begin{array}{l}
s_{1}(t) \\
\bar{s}_{2}^{*}(t)
\end{array}\right]+\left[\begin{array}{c}
n_{1}^{\prime}(t) \\
\tilde{n}_{2}(t)
\end{array}\right]
$$

where the equivalent $S-D$ channel matrix is

$$
\begin{aligned}
& \mathbf{H}\left(q^{-1}, q\right) \\
& \triangleq\left[\begin{array}{ll}
\sqrt{\frac{E_{s} E_{r}}{K_{1}}} g_{1}\left(q^{-1}\right) h_{1}\left(q^{-1}\right) & \sqrt{\frac{E_{s} E_{r}}{K_{2}}} g_{2}\left(q^{-1}\right) h_{2}^{*}(q) \\
-\sqrt{\frac{E_{s} E_{r}}{K_{2}}} g_{2}^{*}(q) h_{2}\left(q^{-1}\right) & \sqrt{\frac{E_{s} E_{r}}{K_{1}}} g_{1}^{*}(q) h_{1}^{*}(q)
\end{array}\right] .
\end{aligned}
$$

Note that the special case of flat-fading $S-R$ and $R-D$ channels and $K_{1}=K_{2}=K$ corresponds to the channel matrix

$$
\mathbf{H}=\sqrt{\frac{E_{s} E_{r}}{K}}\left[\begin{array}{cc}
g_{1} h_{1} & g_{2} h_{2}^{*} \\
-g_{2}^{*} h_{2} & g_{1}^{*} h_{1}^{*}
\end{array}\right]
$$

which has an Alamouti [17] structure, as observed in [8].

Assuming perfect CSI at the receiver, it can process $\mathbf{y}(t)$ [given by (17)] with the space-time filter $\mathbf{H}^{H}\left(q^{-1}, q\right)$ (as in [13]) followed by a whitening filter. Due to the orthogonality of $\mathbf{H}\left(q^{-1}, q\right)$, we have

$$
\begin{aligned}
\mathbf{H}^{H} & \left(q^{-1}, q\right) \mathbf{H}\left(q^{-1}, q\right) \\
& =\left(\sum_{i=1}^{2} \frac{E_{s} E_{r}}{K_{i}} g_{i}^{*}(q) g_{i}\left(q^{-1}\right) h_{i}^{*}(q) h_{i}\left(q^{-1}\right)\right) \mathbf{I} \\
& \triangleq f\left(q^{-1}\right) f^{*}(q) \mathbf{I}
\end{aligned}
$$

where $\mathbf{I}$ is the identity matrix and we define $f\left(q^{-1}\right)$ as the result of the spectral factorization of the coefficient of the identity matrix in (20). Hence

$$
f\left(q^{-1}\right) f^{*}(q)=\left(\sum_{i=1}^{2} \frac{E_{s} E_{r}}{K_{i}} g_{i}^{*}(q) g_{i}\left(q^{-1}\right) h_{i}^{*}(q) h_{i}\left(q^{-1}\right)\right) .
$$

The output vector is given by

$$
\mathbf{z}(t) \triangleq \mathbf{H}^{H}\left(q^{-1}, q\right) \mathbf{y}(t)
$$


whose components can be expressed as

$$
\begin{aligned}
& z_{1}(t)=f\left(q^{-1}\right) f^{*}(q) s_{1}(t)+v_{1}(t) \\
& z_{2}(t)=f\left(q^{-1}\right) f^{*}(q) \bar{s}_{2}^{*}(t)+v_{2}(t)
\end{aligned}
$$

and the output noise $\mathbf{v}(t) \triangleq\left[v_{1}(t) v_{2}(t)\right]^{T}$ has $\operatorname{PSD~}_{\mathbf{v}}\left(q^{-1}, q\right)$ given by

$$
\mathbf{X}_{\mathbf{v}}\left(q^{-1}, q\right)=X\left(q^{-1}, q\right) f\left(q^{-1}\right) f^{*}(q) \mathbf{I}
$$

Thus, $v_{1}(t)$ and $v_{2}(t)$ are independent and the problem of jointly detecting $s_{1}(t)$ and $s_{2}(t)$ from $\mathbf{z}(t)$ decouples: $s_{1}(t)$ is detected from $z_{1}(t)$ and $s_{2}(t)$ from $z_{2}(t)$.

Comparing the filter outputs given by (23) and (24) with the equivalent STBC relations for two antennas in [13], we note two key differences. First, the noise PSD given by (25) contains a term $X\left(q^{-1}, q\right) \neq 1$ due to the noise amplification at the relays. Second, the channel $f\left(q^{-1}\right)$ accounts for the overall effect of the "product" channels $g_{i}\left(q^{-1}\right) h_{i}\left(q^{-1}\right)$, which increases the delay spread (with respect to the individual $g$ or $h$ channels) and impacts performance, as will be shown in Section III. It should also be noted that the noise at the destination is colored (in time), because the $R-D$ channel introduces ISI.

Applying the spectral factorization $X\left(q^{-1}, q\right)=x(q) x\left(q^{-1}\right)$ and, hence, whitening the noise by applying the filter $W\left(q^{-1}, q\right)=\left(1 / x\left(q^{-1}\right) f^{*}(q)\right)$, results in the desired AWGN model

$$
\begin{aligned}
& \tilde{z}_{1}(t)=\frac{f\left(q^{-1}\right)}{x\left(q^{-1}\right)} s_{1}(t)+\tilde{v}_{1}(t) \approx \tilde{c}\left(q^{-1}\right) s_{1}(t)+\tilde{v}_{1}(t) \\
& \tilde{z}_{2}(t)=\frac{f\left(q^{-1}\right)}{x\left(q^{-1}\right)} \bar{s}_{2}^{*}(t)+\tilde{v}_{2}(t) \approx \tilde{c}\left(q^{-1}\right) \bar{s}_{2}^{*}(t)+\tilde{v}_{1}(t)
\end{aligned}
$$

where $\tilde{c}\left(q^{-1}\right)$ denotes the least squares finite impulse response (FIR) approximation of $c\left(q^{-1}\right) \triangleq\left(f\left(q^{-1}\right) / x\left(q^{-1}\right)\right)$, which has an infinite impulse response (IIR), in general. Denoting, respectively, by $L_{f}, L_{x}$, and $L_{\tilde{c}}$ the number of taps in $f\left(q^{-1}\right), x\left(q^{-1}\right)$, and $\tilde{c}\left(q^{-1}\right)$, we set $L_{\tilde{c}}=L_{f}+L_{x}-1$. Due to the least squares design of the FIR approximation, we observed that this choice of $L_{\tilde{c}}$ incurs negligible error compared to the desired filter response.

For the FIR model in (26) and (27), maximum-likelihood sequence estimation (MLSE) can be carried out via a Viterbi-type algorithm [18]. For channels experiencing extensive multipath however (such as the underwater channels), the MLSE detector is prohibitively complex, and a DFE has been shown to yield good results in practice [19].

\section{PERFORMANCE ANALYSIS}

In this section, we provide the performance analysis of the proposed cooperation scheme with DFE at the receiver. We employ the technique described in [20], and, for mathematical tractability, consider the particular scenario in which the
$R-D$ channels have only one multipath component. This case corresponds, for example, to the scenario where propagation occurs via a single surface reflection. Furthermore, we will also assume that all individual links $S-R_{i}$ and $R_{i}-D$ are fading independently (maximum spatial diversity).

First, we note that, due to the orthogonality of the channel matrix $\mathbf{H}\left(q^{-1}, q\right)$, the dual-channel cooperative scheme can be analyzed as two independent single-channel branches: the first being $S-R_{1}-D$ and the second $S-R_{2}-D$. For the same reason, the TR-STBC scheme in [13] was shown to be equivalent to a two-antenna receive diversity system.

Assuming, without loss of generality, communication through the $S-R_{1}-D$ channel, the equivalent received signal at $D$ and time $t$ (after the space-time processing described in the previous section), denoted by $r_{D}(t)$, is given by

$$
\begin{aligned}
r_{D}(t)=\sqrt{\frac{E_{s} E_{r}}{K_{1}}} \sum_{l=-\infty}^{+\infty} s(t-l) h_{1}(l) g_{1} \\
\quad+\sqrt{\frac{E_{r}}{K_{1}}} w_{1}(t) g_{1}+\sqrt{\frac{E_{r}}{K_{2}}} w_{2}(t) g_{2}+n(t)
\end{aligned}
$$

where $g_{1}$ and $g_{2}$ denote, respectively, the (flat) fading realization of the $R_{1}-D$ and $R_{2}-D$ channels and $h_{1}(l)$ the fading realization of the $l$ th path of the $S-R_{1}$ channel. As in the previous section, the noise realizations at both relays are denoted by $w_{1}(t)$ and $w_{2}(t)$ and $n(t)$ is the destination noise.

To decode a desired symbol $s(k)$, a DFE operates on a vector of $N_{1}+1$ received symbols, each in turn given by (28), and $N_{2}$ past decoded symbols $\hat{s}(k-1), \hat{s}(k-2), \ldots, \hat{s}\left(k-N_{2}\right)$. Assuming, without loss of generality, that $s(0)$ is the desired symbol to be detected, the output of the DFE is

$$
\begin{aligned}
I_{0}= & \mathbf{e}^{H}\left(\sqrt{\frac{E_{s} E_{r}}{K_{1}}} s(0) g_{1} \mathbf{h}_{1}(0)+\sqrt{\frac{E_{s} E_{r}}{K_{1}}}\right. \\
& \times \sum_{l=-L_{c}, l \neq 0}^{N_{1}} s(l) g_{1} \mathbf{h}_{1}(l)+\sqrt{\frac{E_{r}}{K_{1}}} \mathbf{w}_{1}(0) g_{1} \\
& \left.+\sqrt{\frac{E_{r}}{K_{2}}} \mathbf{w}_{2}(0) g_{2}+\mathbf{n}(0)\right) \\
+ & \sum_{u=1}^{N_{2}} b^{*}(u) \hat{s}(-u)
\end{aligned}
$$

where $L_{c}+1$ is the number of paths of the overall $S-R_{1}-D$ channel response, and $N_{1}+1$ and $N_{2}$ denote the number of forward and feedback taps, respectively. For a general sequence $a(t)$, we define $\mathbf{a}(k) \triangleq\left[\begin{array}{llll}a(k) & a(k+1) & \ldots & a\left(k+N_{1}\right)\end{array}\right]^{T}$. The vector $\mathbf{e}$, of length $N_{1}+1$, is the forward filter. The feedback coefficients, which operate on the previously decoded symbols, are denoted by $b(1), \ldots, b\left(N_{2}\right)$.

As usual in the literature, we make the key assumption that the previous symbols are decoded perfectly [20]. While this is not the case for low signal-to-noise ratio (SNR), it is a good approximation in the high SNR regime and, furthermore, it is the typical scenario where diversity analysis is performed. Hence, 
the feedback filter completely cancels the interference due to past symbols in (29) and the DFE output becomes

$$
\begin{aligned}
I_{0}=\mathbf{e}^{H} & \left(\sqrt{\frac{E_{s} E_{r}}{K_{1}}} s(0) g_{1} \mathbf{h}_{1}(0)+\sqrt{\frac{E_{s} E_{r}}{K_{1}}} \sum_{l=1}^{N_{1}} s(l) g_{1} \mathbf{h}_{1}(l)\right. \\
& \left.+\sqrt{\frac{E_{r}}{K_{1}}} \mathbf{w}_{1}(0) g_{1}+\sqrt{\frac{E_{r}}{K_{2}}} \mathbf{w}_{2}(0) g_{2}+\mathbf{n}(0)\right)
\end{aligned}
$$

which can be written as

$$
I_{0}=\mathbf{e}^{H}\left(\sqrt{\frac{E_{s} E_{r}}{K_{1}}} s(0) g_{1} \mathbf{h}_{1}(0)+\mathbf{n}_{1}+\mathbf{n}_{2}+\mathbf{n}_{0}\right)
$$

where the destination noise, the ISI due to future symbols, and the overall relay noise are given by, respectively

$$
\begin{aligned}
\mathbf{n}_{0} & =\mathbf{n}(0) \\
\mathbf{n}_{1} & =\sqrt{\frac{E_{s} E_{r}}{K_{1}}} \sum_{l=1}^{N_{1}} s(l) g_{1} \mathbf{h}_{1}(l) \\
\mathbf{n}_{2} & =\sqrt{\frac{E_{r}}{K_{1}}} \mathbf{w}_{1}(0) g_{1}+\sqrt{\frac{E_{r}}{K_{2}}} \mathbf{w}_{2}(0) g_{2} .
\end{aligned}
$$

We now make further simplifying assumptions, also according to [20]. First, we replace the noise and interference covariance matrices by their ensemble averages. Second, we assume that the interference symbols are complex Gaussian with variance 1 . Furthermore, we assume Rayleigh fading, i.e., each tap $k$ in $\mathbf{h}_{1}(0)$ has distribution $\mathcal{C N}\left(0, \sigma_{h_{k}}^{2}\right)$ and $g_{1}$ has distribution $\mathcal{C N}\left(0, \sigma_{g}^{2}\right)$. Under these assumptions, the noise and interference covariance matrices are

$$
\begin{aligned}
\mathbf{K}_{0}= & \mathbf{I} \\
\mathbf{K}_{1}= & \frac{E_{s} E_{r}}{K_{1}} \sigma_{g}^{2} \\
& \times \operatorname{diag}\left[0, \sigma_{h_{0}}^{2}, \sum_{i=0}^{1} \sigma_{h_{i}}^{2}, \ldots, \sum_{i=0}^{L_{c}} \sigma_{h_{i}}^{2}, \ldots, \sum_{i=0}^{L_{c}} \sigma_{h_{i}}^{2}\right]
\end{aligned}
$$

$$
\mathbf{K}_{2}=2 \sigma_{g}^{2} \mathbf{I}
$$

The noise plus interference covariance is

$$
\mathbf{K}=\mathbf{K}_{0}+\mathbf{K}_{1}+\mathbf{K}_{2} \text {. }
$$

From (31), the signal power at the output of the equalizer is

$$
P_{0}=\frac{E_{s} E_{r}}{K_{1}} \mathbf{e}^{H} \mathbf{h}_{1}(0) \mathbf{h}_{1}(0)^{H} \mathbf{e}\left|g_{1}\right|^{2}
$$

and the noise plus interference power is

$$
W_{0}=\mathbf{e}^{H} \mathbf{K e}
$$

Following the same approach as in [20], it can be shown that the optimal choice for the equalizer taps is $\mathbf{e}_{\text {opt }}=\mathbf{K}^{-1} \mathbf{h}_{1}(0) g_{1}$, and the resulting optimal SNR $\xi$ of the equalizer output is

$$
\xi=\frac{E_{s} E_{r}}{K_{1}} \mathbf{h}_{1}(0)^{H} \mathbf{K}^{-1} \mathbf{h}_{1}(0)\left|g_{1}\right|^{2} .
$$

For binary phase-shift keying (BPSK) modulation and given channel realizations $\mathbf{h}_{1}(0)$ and $g_{1}$, the bit error rate (BER) of the system can be estimated as $Q(\sqrt{2 \xi})$, which has to be averaged over the distributions of the channels. A simple upper bound approximation (for high SNR) of the resulting BER is given by the Chernoff bound. Conditioned on $g \triangleq g_{1} / \sigma_{g}$, where $g \sim$ $\mathcal{C N}(0,1)$, the BER is

$$
\begin{aligned}
\left.\mathrm{BER}_{1}\right|_{g} & \leq \frac{1}{2} \prod_{k=0}^{N_{1}}\left(1+\frac{E_{s} E_{r}}{K_{1}[\mathbf{K}]_{k, k}} \sigma_{h_{k}}^{2} \sigma^{2}|g|^{2}\right)^{-1} \\
& \leq \frac{1}{2}\left(1+\frac{E_{s} E_{r}}{K_{1}[\mathbf{K}]_{0,0}} \sigma_{h_{0}}^{2} \sigma_{g}^{2}|g|^{2}\right)^{-1}
\end{aligned}
$$

Defining $\bar{\xi}=\left(E_{s} E_{r} / K_{1}[\mathbf{K}]_{0,0}\right) \sigma_{h_{1,0}}^{2} \sigma_{g}^{2}$ as an average SNR quantity

$$
\left.\mathrm{BER}_{1}\right|_{g} \leq \frac{1}{2}\left(1+\bar{\xi}|g|^{2}\right)^{-1}
$$

Recalling that $|g|^{2}$ has an exponential distribution, the average BER is

$$
\mathrm{BER}_{1} \leq \int_{0}^{\infty} \frac{1}{2}(1+\bar{\xi} x)^{-1} e^{-x} d x
$$

After some manipulations, and noting that $e^{1 / \bar{\xi}} \approx 1$ for high $\bar{\xi}$, we can write $(44)$ as

$$
\mathrm{BER}_{1} \leq \frac{1}{2 \bar{\xi}} \int_{\frac{1}{\bar{\xi}}}^{\infty} \frac{e^{-x}}{x} d x
$$

Recalling that (e.g., see [21]) $\int_{(1 / \bar{\xi})}^{\infty}\left(e^{-x} / x\right) d x=$ $-\operatorname{Ei}(-(1 / \bar{\xi}))$, where Ei denotes the Euler exponential integral, the single-channel BER has the closed-form approximation

$$
\mathrm{BER}_{1} \lesssim-\frac{1}{2 \bar{\xi}} \mathrm{Ei}\left(-\frac{1}{\bar{\xi}}\right)
$$

Thus, assuming perfect symmetry between the channel statistics of the two spatial diversity branches, the desired BER expression is

$$
\mathrm{BER}_{2} \lesssim \frac{1}{4 \bar{\xi}^{2}} \mathrm{Ei}^{2}\left(-\frac{1}{\bar{\xi}}\right)
$$

Because $\operatorname{Ei}(-1 / x), x>0$ has an $O(\log (x))$ behavior for large $x$ [21], the BER behavior with $\bar{\xi}$ is

$$
\mathrm{BER}_{2} \lesssim O\left(\frac{\log \bar{\xi}}{\bar{\xi}}\right)^{2}=O\left(\frac{1}{\bar{\xi}}\right)^{2-2 \frac{\log \log \bar{\xi}}{\log \bar{\xi}}} .
$$


We notice, therefore, that the effective diversity at finite $\bar{\xi}$ is less than 2 by a factor of $2(\log \log \bar{\xi} / \log \bar{\xi})$. For $\bar{\xi} \rightarrow \infty$, the diversity is asymptotically 2 and, thus, full spatial diversity is achieved. The diversity loss at finite SNR can be attributed to the overall $S-R-D$ channel not being of Rayleigh statistics, but of a "product" fading channel instead.

\section{COPING With IMPERFECT RElay SynCHRONIZATION}

The distributed nature of the cooperative communication strategy described in Section II naturally brings up the question of whether the relays need to operate under perfect synchronization. Indeed, this is a common assumption in several recently proposed distributed cooperation strategies [8], [4], [10].

In many practical situations, however, due to different delays between the cooperative nodes and the destination, achieving perfect synchronization can be very difficult [22]. The long sound propagation delays in underwater networks can, therefore, potentially exacerbate this problem.

A direct consequence of imperfect synchronization between relays is the introduction of time dispersion in the channels; this can occur even in frequency flat channels, and is due to imperfect sampling times at the receiver [22], [23]. The TR-DSTBC approach for frequency selective channels can also operate with asynchronous relays, if we assume a known upper bound on the relative transmission delays (to avoid interblock interference).

From the received signal model at the relays given by (4) and (5), the received signal for block $k$ at $D$ assuming a time delay of $\epsilon$ between transmissions from $R_{1}$ and $R_{2}$ is

$$
\begin{aligned}
y_{1}(t)= & \sqrt{\frac{E_{s} E_{r}}{K_{1}}} g_{1}\left(q^{-1}\right) h_{1}\left(q^{-1}\right) s_{1}(t) \\
& +\sqrt{\frac{E_{s} E_{r}}{K_{2}}} g_{2}\left(q^{-1}\right) h_{2}^{*}(q) R_{p_{\epsilon}}\left(q^{-1}, q\right) \bar{s}_{2}^{*}(t)+n_{1}^{\prime}(t) \\
y_{2}(t)= & \sqrt{\frac{E_{s} E_{r}}{K_{1}}} g_{1}\left(q^{-1}\right) h_{1}\left(q^{-1}\right) s_{2}(t) \\
& -\sqrt{\frac{E_{s} E_{r}}{K_{2}}} g_{2}\left(q^{-1}\right) h_{2}^{*}(q) R_{p_{\epsilon}}\left(q^{-1}, q\right) \bar{s}_{1}^{*}(t)+n_{2}^{\prime}(t)
\end{aligned}
$$

where the filter $R_{p_{\epsilon}}\left(q^{-1}, q\right)$ denotes the transmit pulse correlation function for a delay $\epsilon$, with coefficients

$$
R_{p_{\epsilon}}(k)=\left.p(t+\epsilon) * p(-t)\right|_{t=k T}, \quad-\infty<k<\infty
$$

where $p(t)$ denotes the transmit pulse shape, usually a raised cosine pulse [19] of unit energy. Hence, in a perfectly synchronized scenario, $R_{p_{0}}\left(q^{-1}, q\right)=1$.

Clearly, from (49) and (50), the imperfect synchronization increases the dispersion of the $R_{2}-D$ channel. However, defining an equivalent channel $h_{2}^{\prime}\left(q^{-1}\right) \triangleq R_{p_{\epsilon}}\left(q^{-1}, q\right) h_{2}\left(q^{-1}\right)$, an identical form of the signal model in (17) is obtained, with $h_{2}^{\prime}\left(q^{-1}\right)$ replacing $h_{2}\left(q^{-1}\right)$. Thus, the TR-DSTBC formulation can conveniently address the case of asynchronous relays by simple generalization of the channel model.

In Section VII, experimental results for asynchronous relays (a consequence of the relays being deployed over different depths) are reported.

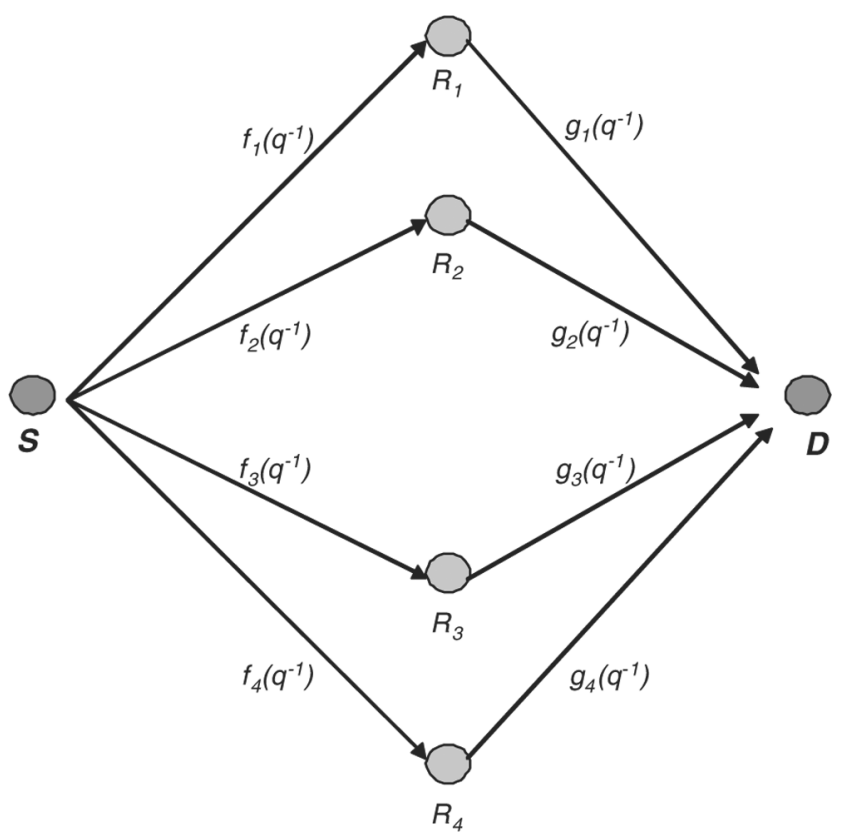

Fig. 2. Two-hop cooperative network with four relays.

\section{InCREASING Spatial Diversity With More Relays}

The key property that allows signal decoupling at receiver for the two-relay scheme presented in Section II is the orthogonality of the Alamouti STBC. It allows two signal streams to be transmitted via two relays over two blocks of time, and, hence, exhibits full rate of one.

Unfortunately, no full rate orthogonal STBC exists (for complex modulation) for more than two antennas [7]. In this section, we present a generalization of the two relay scheme for two hops presented earlier to arbitrary number of relays based on rate $1 / 2$ orthogonal STBCs [7], denoted by TR-1/2. For simplicity, we describe the TR-1/2 scheme for the case of four relays depicted in Fig. 2. It can be readily extended to more relays combining the distributed signaling approach described here with the rate $1 / 2$ STBC structure in [7].

The information stream $d(t)$ is now divided into four equal length blocks $d_{1}(t), \ldots, d_{4}(t)$. The source transmission occurs over eight blocks: in the first four blocks,

$$
s_{k}(t)=\sqrt{E_{s}} d_{k}(t), \quad k=1, \ldots, 4
$$

are transmitted; in the last four blocks,

$$
s_{k}(t)=\sqrt{E_{s}} \bar{d}_{k-4}^{*}(t), \quad k=5, \ldots, 8
$$

are sent. Note that the blocks of the second half transmission are the time-reversed conjugates of the first half blocks. Hence, the rate of the scheme is $1 / 2$.

The received signals at relay $i, i=1, \ldots, 4$ and block $k$, $k, i=1, \ldots, 8$, are

$$
r_{i, k}(t)=\sqrt{E_{s}} h_{i}\left(q^{-1}\right) s_{k}(t)+w_{i, k}(t), \quad t=1, \ldots, N
$$

where, as before, the $h_{i}\left(q^{-1}\right)$ denotes the channel coefficients between source and relay $i$ and $w_{i, k}(t)$ is AWGN. The processing performed by each relay over its received blocks is dictated by the underlying rate $1 / 2$ STBC structure. In general, for 
symbols $x_{i}, i=1, \ldots, 4$, from a complex alphabet, the rate $1 / 2$ $\mathrm{STBC}$ is

$$
\mathbf{S}=\left[\begin{array}{c}
S \\
S^{*}
\end{array}\right], \quad \text { where } S=\left[\begin{array}{cccc}
x_{1} & x_{2} & x_{3} & x_{4} \\
-x_{2} & x_{1} & -x_{4} & x_{3} \\
-x_{3} & x_{4} & x_{1} & -x_{2} \\
-x_{4} & -x_{3} & x_{2} & x_{1}
\end{array}\right] .
$$

Thus, if each relay corresponds to a different column of $\mathbf{S}$, and each row to a different time block, the relay transmissions can be represented by the matrix shown in (56) at the bottom of the page, where each column $\mathbf{u}_{i}(t)=\left[u_{i, 1}(t), \ldots, u_{i, 8}(t)\right]^{T}$ consists of the transmission of eight blocks, according to (56) and (54).

Denoting by $g_{i}\left(q^{-1}\right)$ the channels from relay $i$ to the destination, the received signal at the destination (after time reversing and conjugating the second-half received blocks) is

$$
\begin{aligned}
& \mathbf{y}(t)=\left[\begin{array}{cccc}
f_{1}\left(q^{-1}\right) & f_{2}\left(q^{-1}\right) & f_{3}\left(q^{-1}\right) & f_{4}\left(q^{-1}\right) \\
f_{2}\left(q^{-1}\right) & -f_{1}\left(q^{-1}\right) & f_{4}\left(q^{-1}\right) & -f_{3}\left(q^{-1}\right) \\
-f_{3}\left(q^{-1}\right) & f_{4}\left(q^{-1}\right) & f_{1}\left(q^{-1}\right) & -f_{2}\left(q^{-1}\right) \\
-f_{4}\left(q^{-1}\right) & -f_{3}\left(q^{-1}\right) & f_{2}\left(q^{-1}\right) & f_{1}\left(q^{-1}\right) \\
f_{1}^{*}(q) & f_{2}^{*}(q) & f_{3}^{*}(q) & f_{4}^{*}(q) \\
f_{2}^{*}(q) & -f_{1}^{*}(q) & f_{4}^{*}(q) & -f_{3}^{*}(q) \\
-f_{3}^{*}(q) & f_{4}^{*}(q) & f_{1}^{*}(q) & -f_{2}^{*}(q) \\
-f_{4}^{*}(q) & -f_{3}^{*}(q) & f_{2}^{*}(q) & f_{1}^{*}(q)
\end{array}\right] \\
& \times\left[\begin{array}{l}
d_{1}(t) \\
d_{2}(t) \\
d_{3}(t) \\
d_{4}(t)
\end{array}\right] \\
& +\left[\begin{array}{c}
\sum_{i=1}^{4} w_{i, 1}(t) g_{i}\left(q^{-1}\right)+\eta_{1}(t) \\
\vdots \\
\sum_{i=1}^{4} w_{i, 4}(t) g_{i}\left(q^{-1}\right)+\eta_{4}(t) \\
\sum_{i=1}^{4} \bar{w}_{i, 5}^{*}(t) g_{i}\left(q^{-1}\right)+\bar{\eta}_{5}^{*}(t) \\
\vdots \\
\sum_{i=1}^{4} \bar{w}_{i, 8}^{*}(t) g_{i}\left(q^{-1}\right)+\bar{\eta}_{8}^{*}(t)
\end{array}\right]
\end{aligned}
$$

where $\eta_{k}(t)$ is additive white noise at the receiver and $f_{i}\left(q^{-1}\right)=\sqrt{\left(E_{s} E_{r} / K_{i}\right)} h_{i}\left(q^{-1}\right) g_{i}\left(q^{-1}\right)$ is the overall channel $S-R_{i}-D$.

Just as in the two-relay case, we can define the channel matrix

$$
\begin{aligned}
& \mathbf{H}\left(q^{-1}, q\right) \\
& \triangleq\left[\begin{array}{cccc}
f_{1}\left(q^{-1}\right) & f_{2}\left(q^{-1}\right) & f_{3}\left(q^{-1}\right) & f_{4}\left(q^{-1}\right) \\
f_{2}\left(q^{-1}\right) & -f_{1}\left(q^{-1}\right) & f_{4}\left(q^{-1}\right) & -f_{3}\left(q^{-1}\right) \\
-f_{3}\left(q^{-1}\right) & f_{4}\left(q^{-1}\right) & f_{1}\left(q^{-1}\right) & -f_{2}\left(q^{-1}\right) \\
-f_{4}\left(q^{-1}\right) & -f_{3}\left(q^{-1}\right) & f_{2}\left(q^{-1}\right) & f_{1}\left(q^{-1}\right) \\
f_{1}^{*}(q) & f_{2}^{*}(q) & f_{3}^{*}(q) & f_{4}^{*}(q) \\
f_{2}^{*}(q) & -f_{1}^{*}(q) & f_{4}^{*}(q) & -f_{3}^{*}(q) \\
-f_{3}^{*}(q) & f_{4}^{*}(q) & f_{1}^{*}(q) & -f_{2}^{*}(q) \\
-f_{4}^{*}(q) & -f_{3}^{*}(q) & f_{2}^{*}(q) & f_{1}^{*}(q)
\end{array}\right]
\end{aligned}
$$

which satisfies

$$
\begin{aligned}
\mathbf{H}^{H} & \left(q^{-1}, q\right) \mathbf{H}\left(q^{-1}, q\right) \\
& =\left(2 \sum_{i=1}^{4} \sqrt{\frac{E_{s} E_{r}}{K_{i}}} g_{i}\left(q^{-1}\right) g_{i}^{*}(q) h_{i}\left(q^{-1}\right) h_{i}^{*}(q)\right) \mathbf{I} \\
& \triangleq f\left(q^{-1}\right) f^{*}(q) \mathbf{I}
\end{aligned}
$$

and, hence, is orthogonal.

The output vector is given by

$$
\mathbf{z}(t) \triangleq \mathbf{H}^{H}\left(q^{-1}, q\right) \mathbf{y}(t)
$$

consisting of four blocks, each one given by

$$
z_{i}(t)=f\left(q^{-1}\right) f^{*}(q) d_{i}(t)+v_{i}(t), \quad i=1, \ldots, 4
$$

and the output noise $\mathbf{v}(t) \triangleq\left[v_{1}(t), \ldots, v_{4}(t)\right]^{T}$ has PSD

$$
X_{\mathbf{v}}\left(q^{-1}, q\right)=X\left(q^{-1}, q\right) f\left(q^{-1}\right) f^{*}(q) \mathbf{I} \text {. }
$$

Hence, $v_{1}(t), \ldots, v_{4}(t)$ are independent and the problem of jointly detecting $d_{i}(t), i=1, \ldots, 4$, from $\mathbf{z}(t)$ again decouples, just like in the two-relay case.

$$
U \triangleq\left[\begin{array}{l}
\mathbf{u}_{1}(t) \quad \mathbf{u}_{2}(t) \quad \mathbf{u}_{3}(t) \quad \mathbf{u}_{4}(t)
\end{array}\right] \triangleq\left[\begin{array}{cccc}
\sqrt{\frac{E_{r}}{K_{1}}} r_{1,1}(t) & \sqrt{\frac{E_{r}}{K_{2}}} r_{2,2}(t) & \sqrt{\frac{E_{r}}{K_{3}}} r_{3,3}(t) & \sqrt{\frac{E_{r}}{K_{4}}} r_{4,4}(t) \\
-\sqrt{\frac{E_{r}}{K_{1}}} r_{1,2}(t) & \sqrt{\frac{E_{r}}{K_{2}}} r_{2,1}(t) & -\sqrt{\frac{E_{r}}{K_{3}}} r_{3,4}(t) & \sqrt{\frac{E_{r}}{K_{4}}} r_{4,3}(t) \\
-\sqrt{\frac{E_{r}}{K_{1}}} r_{1,3}(t) & \sqrt{\frac{E_{r}}{K_{2}}} r_{2,4}(t) & \sqrt{\frac{E_{r}}{K_{3}}} r_{3,1}(t) & -\sqrt{\frac{E_{r}}{K_{4}}} r_{4,2}(t) \\
-\sqrt{\frac{E_{r}}{K_{1}}} r_{1,4}(t) & -\sqrt{\frac{E_{r}}{K_{2}}} r_{2,3}(t) & \sqrt{\frac{E_{r}}{K_{3}}} r_{3,2}(t) & \sqrt{\frac{E_{r}}{K_{4}}} r_{4,1}(t) \\
\sqrt{\frac{E_{r}}{K_{1}}} r_{1,5}(t) & \sqrt{\frac{E_{r}}{K_{2}}} r_{2,6}(t) & \sqrt{\frac{E_{r}}{K_{3}}} r_{3,7}(t) & \sqrt{\frac{E_{r}}{K_{4}}} r_{4,8}(t) \\
-\sqrt{\frac{E_{r}}{K_{1}}} r_{1,6}(t) & \sqrt{\frac{E_{r}}{K_{2}}} r_{2,5}(t) & -\sqrt{\frac{E_{r}}{K_{3}}} r_{3,8}(t) & \sqrt{\frac{E_{r}}{K_{4}}} r_{4,7}(t) \\
-\sqrt{\frac{E_{r}}{K_{1}}} r_{1,7}(t) & \sqrt{\frac{E_{r}}{K_{2}}} r_{2,8}(t) & \sqrt{\frac{E_{r}}{K_{3}}} r_{3,5}(t) & -\sqrt{\frac{E_{r}}{K_{4}}} r_{4,6}(t) \\
-\sqrt{\frac{E_{r}}{K_{1}}} r_{1,8}(t) & -\sqrt{\frac{E_{r}}{K_{2}}} r_{2,7}(t) & \sqrt{\frac{E_{r}}{K_{3}}} r_{3,6}(t) & \sqrt{\frac{E_{r}}{K_{4}}} r_{4,5}(t)
\end{array}\right]
$$


It turns out that [7]: 1) a rate 1/2 complex orthogonal design exists for any size $2 n \times n$ where $n$ is a power of two, and 2) an orthogonal design of size $l \times n$ where $l<n$ can be obtained by deleting $n-l$ columns of the $2 n \times n$ design. Hence, the TR-1/2 scheme can be generalized to any number of relays in a straightforward fashion.

\section{Underwater ChanNel Model}

We adopt a ray-based model similar to [15] and [16] to model the multipath sound propagation. The main difference in our model is that we assume that the multipath components are fading. We consider a quasi-static fading model, in which the channel is constant within a fixed duration and changes to an independent realization over the next time frame. We analyze the effects of a slowly time-varying channel within the frame as well as nonperfect CSI via simulations in the next section.

A given multipath arrival $p$ is characterized by its mean magnitude gain $\alpha_{p}$ and delay $t_{p}$. These quantities are dependent on the path length $l_{p}$, which in turn is a function of the given range $R$. The path magnitude gain is given by

$$
\alpha_{p}=\frac{\Gamma_{p}}{\sqrt{A\left(l_{p}\right)}}
$$

where $\Gamma_{p}=(1 / \sqrt{2})^{r_{p}}$ is the total reflection loss at the bottom and surface and $r_{p}$ is the number of reflections for path $p$. The acoustic propagation loss, represented by $A\left(l_{p}\right)$, is given by Thorp's formula

$$
A\left(l_{p}\right)=l_{p}^{k}\left[a\left(f_{c}\right)\right]^{l_{p}}
$$

where $k=1.5$ for practical spreading, $f_{c}$ is the carrier frequency, and the absorption coefficient $a\left(f_{c}\right)$ (in decibels per kilometer) is given by Thorp's formula

$10 \log a\left(l_{p}\right)=\frac{0.11 f_{c}^{2}}{1+f_{c}^{2}}+\frac{44 f_{c}^{2}}{4100+f_{c}^{2}}+2.75 .10^{-4} f_{c}^{2}+0.003$.

Finally, the path delay is given by $t_{p}=l_{p} / c$, where $c=$ $1500 \mathrm{~m} / \mathrm{s}$ is the speed of sound.

Fig. 3 shows the channel path delays and magnitudes for a distance of $3 \mathrm{~km}$ between the transmitter and the receiver, $f_{c}=$ $15 \mathrm{kHz}$, and depth of $75 \mathrm{~m}$.

\section{Simulation Results}

\section{A. Computer Channel Simulations}

For simulation purposes, we consider an underwater network with $S-D$ distance of $6 \mathrm{~km}$, carrier frequency $f_{c}=15 \mathrm{kHz}$, and channel depth of $75 \mathrm{~m}$. The total energy spent by the relays was set to be equal to the energy spent by the source, i.e., $E_{r}=E_{s} / R$, where $R$ is the number of relays. The relay normalizing factors are equal to 1 . Three communication strategies are compared: single hop, where the source communicates directly to the destination and cooperative (two hops) with either two or four relays, as described in Sections II and V.

Our first simulation scenario compares performance of the MLSE and DFE to justify the use of the latter for signal detection in our multihop cooperative setting. The modulation is BPSK with a symbol duration of $T=2.5 \mathrm{~ms}$ and, therefore, a data rate of $400 \mathrm{~b} / \mathrm{s}$.

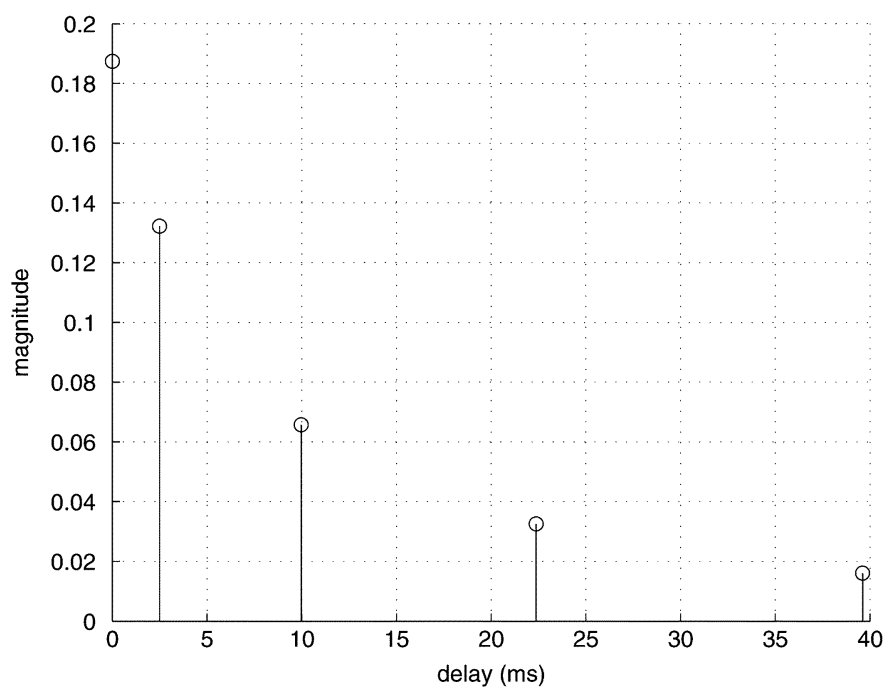

Fig. 3. Two-hop channel profile: $d=3 \mathrm{~km}$ (range per hop), $f_{c}=15 \mathrm{kHz}$, depth $=75 \mathrm{~m}$.

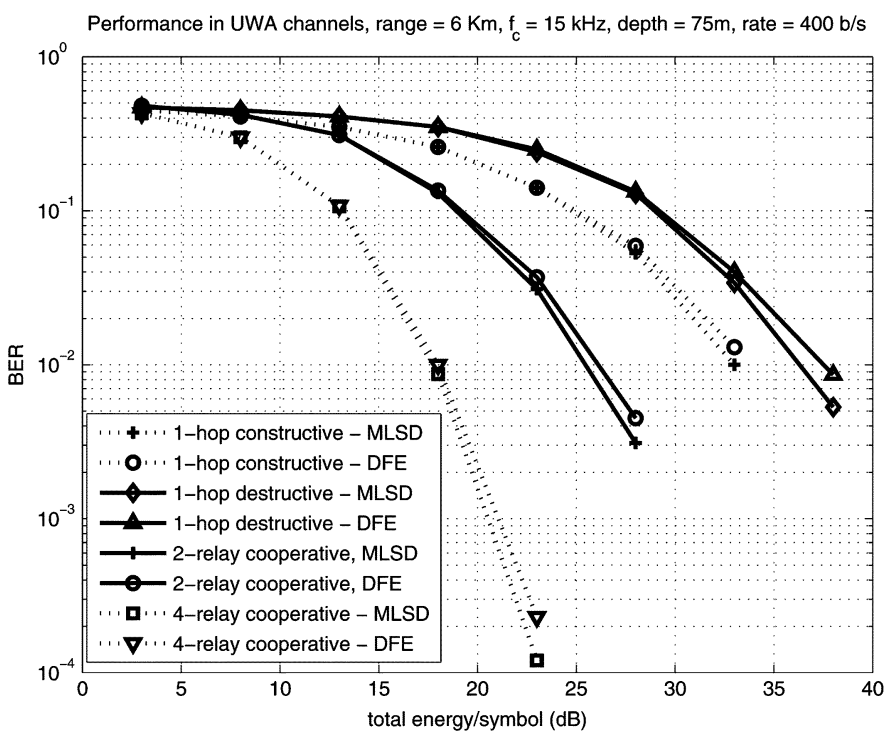

Fig. 4. Two-hop cooperation performance.

This results in a channel with two taps per hop in the cooperative strategy, and three taps for the direct communication approach. In the latter case, multipath arrivals can occur within a fraction of a symbol time. In this case, these arrivals can add up constructively [16], and thus result in a stronger path, or destructively [24], weakening the resulting overall multipath component. We consider both scenarios separately here.

Fig. 4 shows the performance of the MLSD and DFE approaches for the cooperative and single-hop strategies. For the DFE, the number of feedforward and feedback filter taps is $N_{1}+1=15$ and $N_{2}=L_{c}$, respectively, where $L_{c}+1$ is the number of taps of the overall $S-D$ channel. It is clear for all cases considered that the DFE performance is very close to MLSD. Furthermore, the results highlight the significant performance improvement introduced by cooperation. The two main reasons are as follows: 1) multihoping gain, since the overall attenuation suffered by the signal is less severe, and 2) spatial diversity gain, which is due to the spatial diversity inherently 


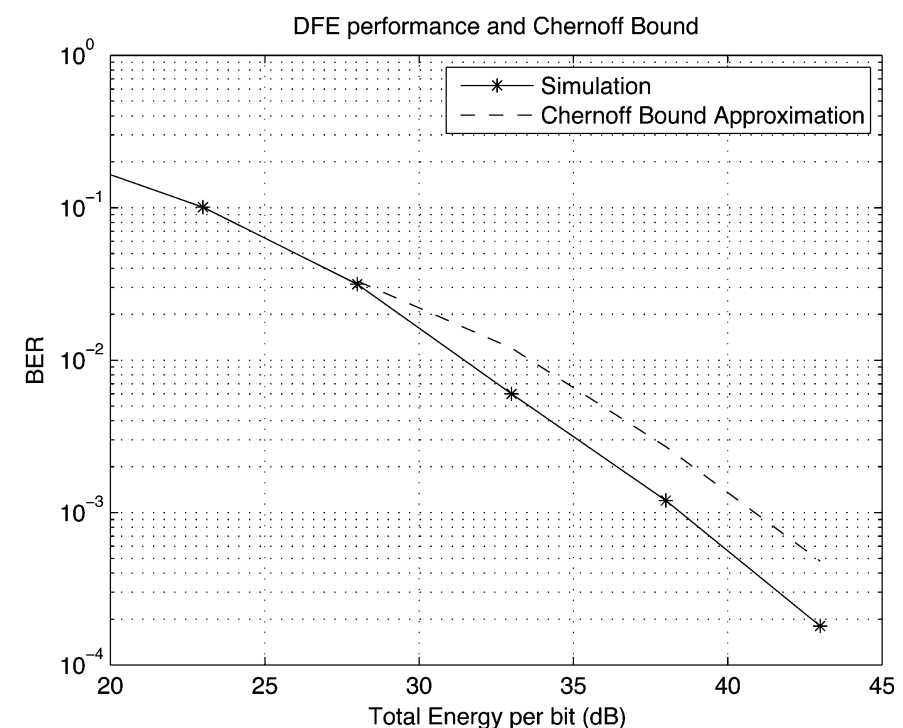

Fig. 5. Chernoff bound for two-hop cooperation.

available in the distributed network, that is exploited via distributed space-time processing.

Note that, although both the single-hop and two-hop cooperative communication approaches yield diversity gain through multipath combining, only the latter provides extra gains through spatial diversity. This explains the larger decay slope of the error probability curves for the cooperative schemes.

Finally, we note that increasing the number of cooperating relays further increases the available spatial diversity in the system and, hence, further improves the performance. This can also be verified for the four-relay case in Fig. 4, which employs the TR-1/2 scheme.

Fig. 5 shows the Chernoff bound in (47) with $\gamma=1$, compared with the simulation performance. Better approximations can be obtained by fine-tuning $\gamma$ via simulations [20]. It can be observed that, at high SNR, the bound indeed confirms the decay order predicted by (48).

1) Sensitivity to Imperfect CSI: In a practical scenario, the receiver does not have perfect knowledge of the channel. In our current framework, CSI at the receiver can be obtained via known training symbols sent from the relays (to estimate the $R_{i}-D$ channels) and from the source (to estimate the $S-R_{i}-D$ channels, such as in [8]).

Due to the presence of channel estimation errors, a degradation in the idealized performance results is naturally expected. In this section, we assess, via simulations, the performance of the receiver employing a DFE under different imperfect CSI conditions.

We model the channel estimation error as a random error matrix that is added to the true channel matrix $\mathbf{H}\left(q^{-1}, q\right)$. The estimated channel matrix $\hat{\mathbf{H}}\left(q^{-1}, q\right)$ is given by

$$
\hat{\mathbf{H}}\left(q^{-1}, q\right)=\mathbf{H}\left(q^{-1}, q\right)+\mathbf{E}\left(q^{-1}, q\right)
$$

with $\mathbf{E}\left(q^{-1}, q\right)$ expressed as

$$
\mathbf{E}\left(q^{-1}, q\right)=\left[\begin{array}{ll}
E_{11}\left(q^{-1}, q\right) & E_{12}\left(q^{-1}, q\right) \\
E_{21}\left(q^{-1}, q\right) & E_{22}\left(q^{-1}, q\right)
\end{array}\right]
$$

where the terms $E_{i j}\left(q^{-1}, q\right)$ in $\mathbf{E}\left(q^{-1}, q\right)$ depend on the estimation errors in the entries of $\mathbf{H}\left(q^{-1}, q\right)$.

We now find expressions for the terms of the matrix $\mathbf{E}\left(q^{-1}, q\right)$. First, in terms of the actual channel estimates $\hat{g}_{i}\left(q^{-1}, q\right)$ and $\hat{h}_{i}\left(q^{-1}, q\right)$, we can write

$$
\hat{\mathbf{H}}\left(q^{-1}, q\right) \triangleq\left[\begin{array}{cc}
\hat{g}_{1}\left(q^{-1}\right) \hat{h}_{1}\left(q^{-1}\right) & \hat{g}_{2}\left(q^{-1}\right) \hat{h}_{2}^{*}(q) \\
-\hat{g}_{2}^{*}(q) \hat{h}_{2}\left(q^{-1}\right) & \hat{g}_{1}^{*}(q) \hat{h}_{1}^{*}(q)
\end{array}\right]
$$

where the constant terms $\sqrt{\left(E_{s} E_{r} / K_{i}\right)}$ are now incorporated into the channels, for notational simplicity. Denoting by $e_{h_{i}}\left(q^{-1}\right)$ and $e_{g_{i}}\left(q^{-1}\right)$ the respective estimation errors in $h_{i}\left(q^{-1}\right)$ and $g_{i}\left(q^{-1}\right)$, the channel estimates are given by

$$
\begin{aligned}
\hat{h}_{i}\left(q^{-1}\right) & =h_{i}\left(q^{-1}\right)+e_{h_{i}}\left(q^{-1}\right) \\
\hat{g}_{i}\left(q^{-1}\right) & =g_{i}\left(q^{-1}\right)+e_{g_{i}}\left(q^{-1}\right) .
\end{aligned}
$$

Hence, we can write

$$
\begin{aligned}
E_{11}\left(q^{-1}, q\right)= & e_{h_{1}}\left(q^{-1}\right)\left(e_{g_{1}}\left(q^{-1}\right)+g_{1}\left(q^{-1}\right)\right) \\
& +e_{g_{1}}\left(q^{-1}\right) h_{1}\left(q^{-1}\right) \\
E_{12}\left(q^{-1}, q\right)= & e_{h_{2}}^{*}(q)\left(e_{g_{2}}\left(q^{-1}\right)+g_{2}\left(q^{-1}\right)\right) \\
& +e_{g_{2}}\left(q^{-1}\right) h_{2}^{*}(q) \\
E_{21}\left(q^{-1}, q\right)= & -e_{h_{2}}\left(q^{-1}\right)\left(e_{g_{2}}^{*}(q)+g_{2}^{*}(q)\right) \\
& -e_{g_{2}}^{*}(q) h_{2}\left(q^{-1}\right) \\
E_{22}\left(q^{-1}, q\right)= & e_{h_{1}}^{*}(q)\left(e_{g_{2}}\left(q^{-1}\right)+g_{1}^{*}(q)\right) \\
& +e_{g_{1}}^{*}(q) h_{1}^{*}(q) .
\end{aligned}
$$

The receiver processing in (22) is now performed using the channel estimate $\hat{\mathbf{H}}\left(q^{-1}, q\right)$. From (17) and (22)

$$
\begin{aligned}
\hat{\mathbf{z}}(t)= & \hat{\mathbf{H}}^{H}\left(q^{-1}, q\right) \mathbf{y}(t) \\
= & \mathbf{z}(t)+\mathbf{E}^{H}\left(q^{-1}, q\right) \mathbf{H}\left(q^{-1}, q\right)\left[\begin{array}{c}
s_{1}(t) \\
\bar{s}_{2}^{*}(t)
\end{array}\right] \\
& +\mathbf{E}^{H}\left(q^{-1}, q\right)\left[\begin{array}{l}
\eta_{1}^{\prime}(t) \\
\tilde{\eta}_{2}(t)
\end{array}\right] .
\end{aligned}
$$

Assuming $s_{1}(t)$ is to be detected, we notice from (75) that the imperfect CSI incurs not only noise enhancement of the decision statistic, but also self and interblock interference from $s_{1}(t)$ and $s_{2}(t)$, respectively.

Fig. 6 shows the performance sensitivity of the two-relay scheme for imperfect CSI. The estimation error in each path $p$ for all channels is assumed to be complex Gaussian with variance $\sigma_{e_{p}}^{2}=\sigma_{e}^{2} \sigma_{p}^{2}$, where $\sigma_{p}^{2}$ is the average square magnitude of path $p$. For $\sigma_{e}^{2}=-20 \mathrm{~dB}$, the degradation is indistinguishable from the perfect CSI case and it deteriorates rather gracefully with increasing $\sigma_{e}^{2}$. As expected, for large estimation errors, the system cannot take full advantage of the spatial diversity, and significant performance deterioration occurs.

2) Sensitivity to Channel Time Variations: To investigate the performance of the system with channel time variations, we assume a first-order time-varying model for each path of the overall channel $f\left(q^{-1}\right)$ in the model derived in (23) and (24). Hence, the receiver filter outputs are

$$
\begin{aligned}
& z_{1}(t)=f\left(q^{-1}, t\right) f^{*}(q, t) s_{1}(t)+v_{1}(t) \\
& z_{2}(t)=f\left(q^{-1}, t\right) f^{*}(q, t) \bar{s}_{2}^{*}(t)+v_{2}(t) .
\end{aligned}
$$




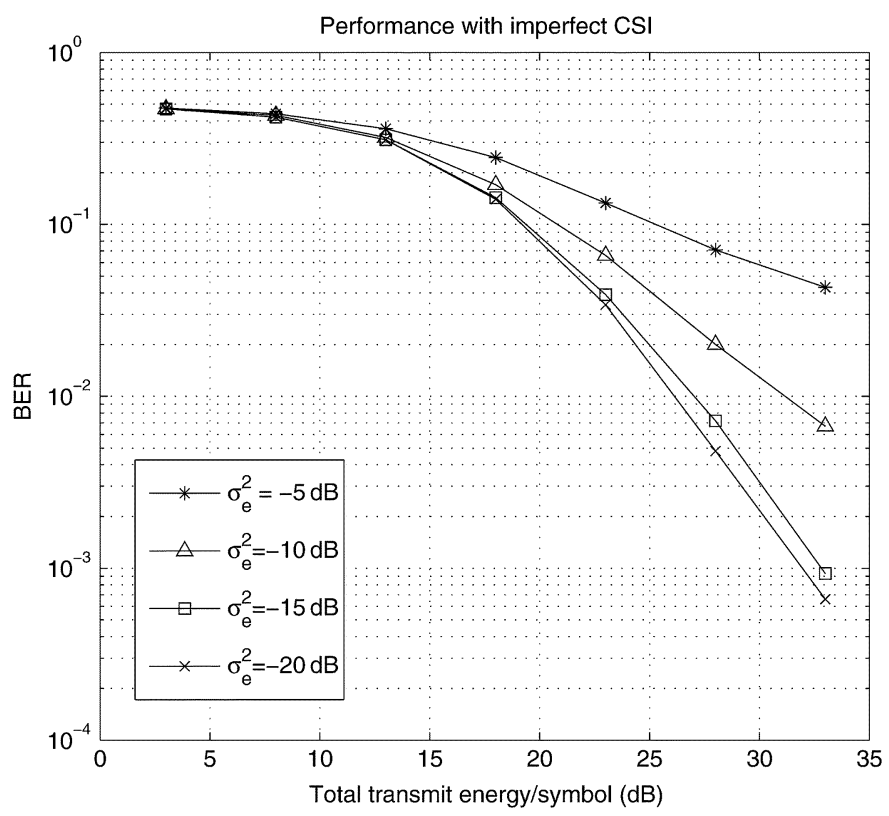

Fig. 6. Two-hop cooperation performance: sensitivity to channel estimation errors.

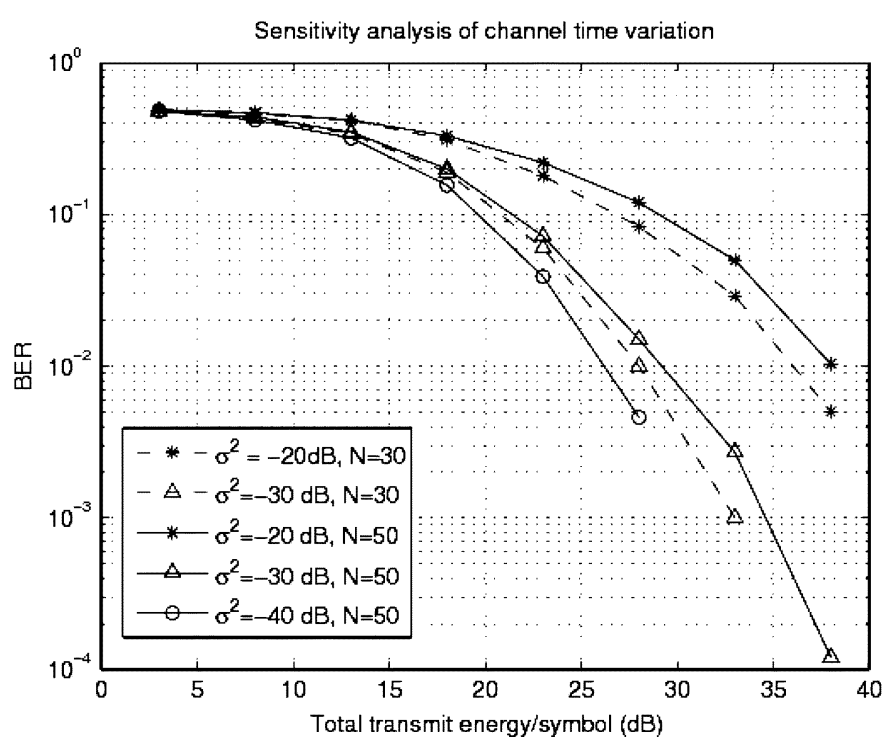

Fig. 7. Two-hop cooperation performance: sensitivity to channel time variations.

A first-order autoregressive (AR) model is assumed for each of the tap coefficients of the filter $f\left(q^{-1}, t\right)$. A similar approach to model channel time-variation characteristics was also employed in [25]. Each time-varying tap $f_{i}(t)$ is modeled as

$$
f_{i}(t)=\sigma f_{i}(t-1)+\sqrt{1-\sigma^{2}} \epsilon(t)
$$

where $\epsilon(t)$ is a complex white Gaussian innovation process of unit variance. For a symbol rate $f_{s}$, we can relate the Doppler spread and the AR parameter $\sigma$ as [25]

$$
\sigma=2-\cos \left(w_{d} / 2\right)-\sqrt{\cos ^{2}\left(w_{d} / 2\right)-4 \cos \left(w_{d} / 2\right)+3} .
$$

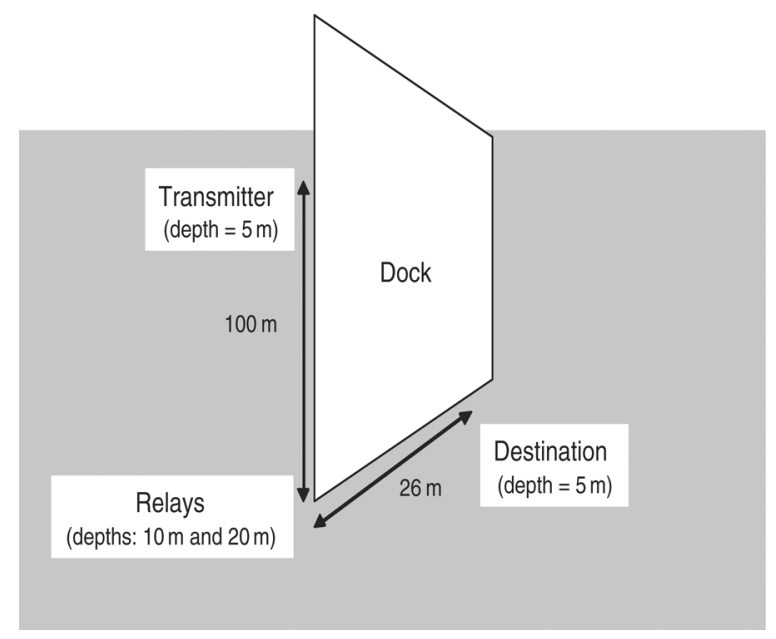

Fig. 8. Experimental setup.

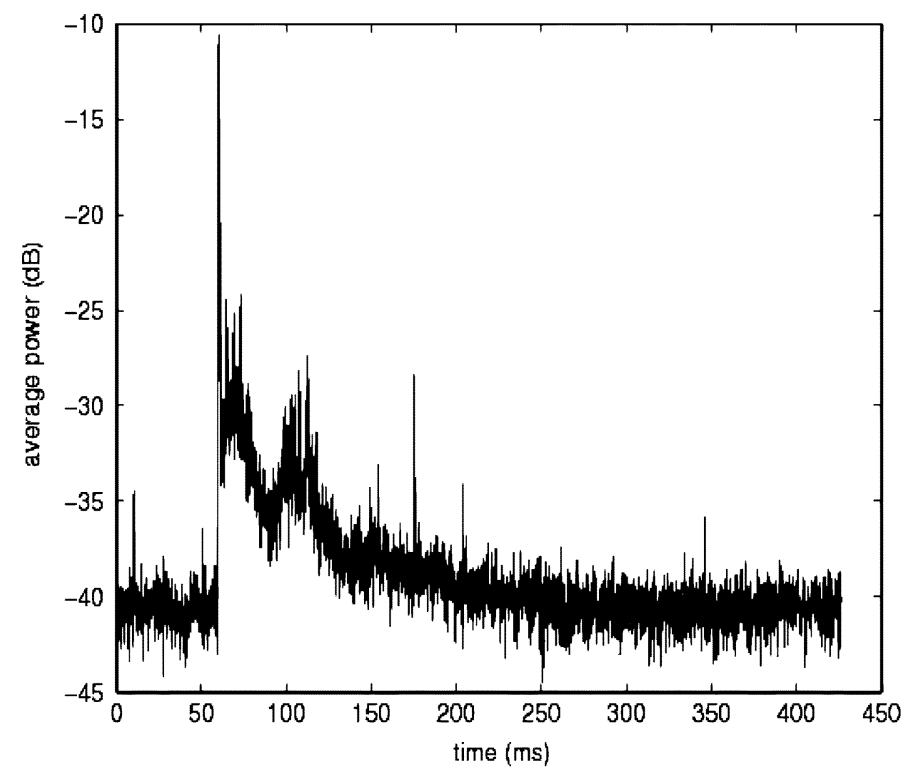

Fig. 9. Source-relay average channel response.

Fig. 7 shows the performance of the scheme under several time-varying conditions, controlled by the parameter $\sigma$. Two types of frame length were used: 50 and 30 symbols. For $N=$ 50 , the time variations accumulated across the frame incurs a slightly more severe degradation compared to a $N=30$ frame. Furthermore, for $N=50$ and $\sigma=-40 \mathrm{~dB}$, the degradation is indistinguishable from the case with no time variations. For both frame length sizes considered, most of the performance gains are still observed for channel variations in the range from $\sigma=-40 \mathrm{~dB}$ to $-30 \mathrm{~dB}$.

\section{B. Experimental Results}

To further verify the potential gains achieved via cooperation, the proposed two-relay protocol was compared with a direct $S-$ $D$ communication approach with measured channel data. The data acquisition experiments were performed at the Woods Hole Oceanographic Institution, Woods Hole, MA, in June 2006.

The experimental setup is depicted in Fig. 8. The carrier frequency was $12 \mathrm{kHz}$. The source transmitter and destination 


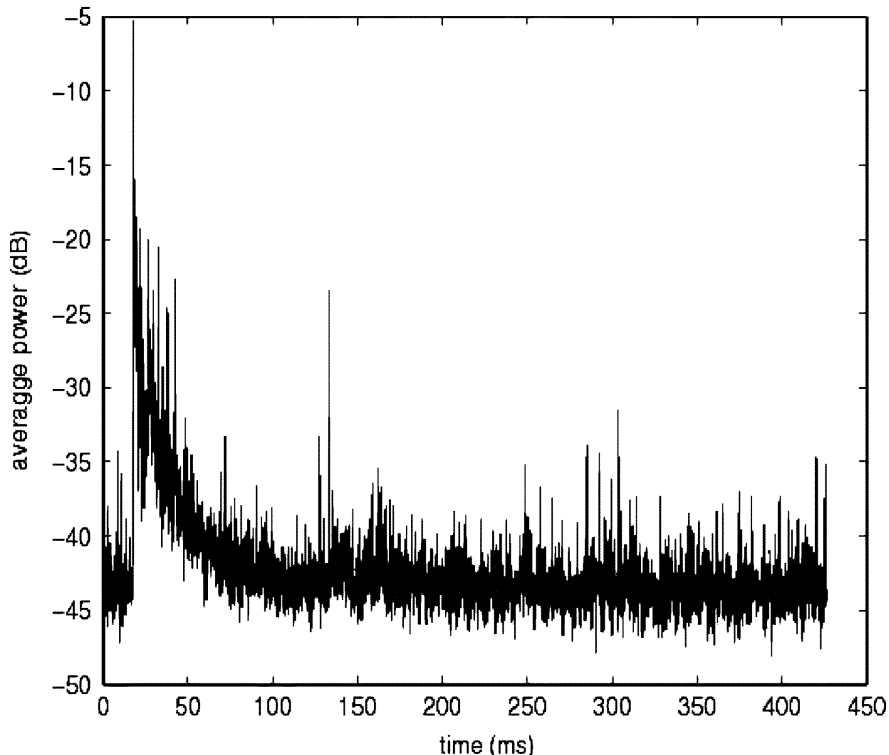

Fig. 10. Relay-destination average channel response.

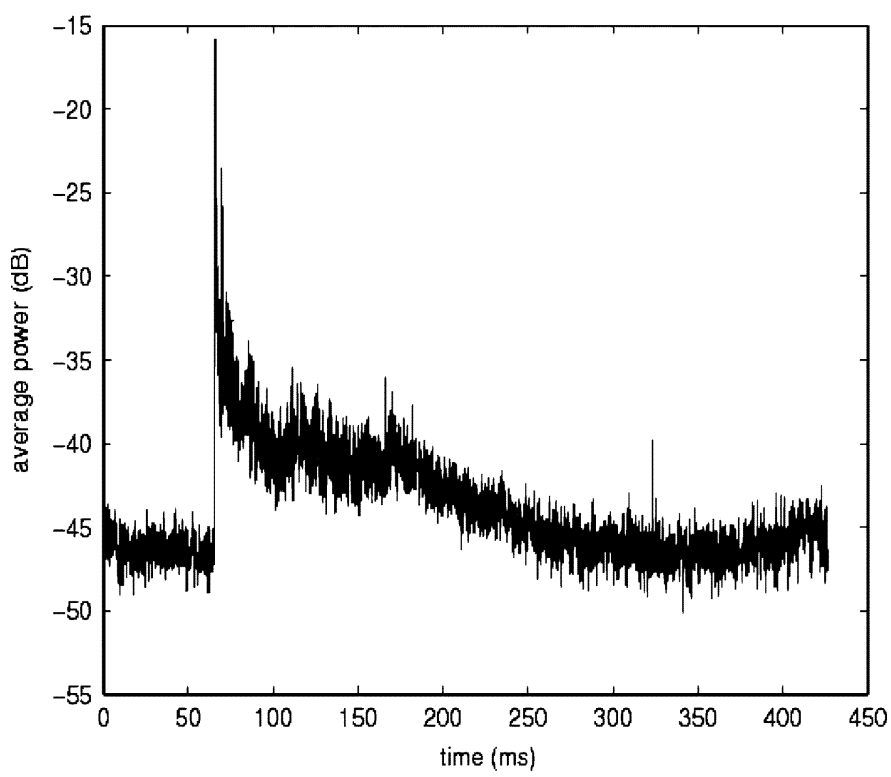

Fig. 11. Source-destination average channel response.

hydrophone were deployed in about 5-m-deep water, and the two hydrophones at the relays were placed in 10- and 20-m-deep water. All hydrophones were omnidirectional. The probe data were maximum-length shift pseudonoise sequences of unshaped (rectangular) pulses.

Figs. 9-11 show the averaged channel response over an ensemble of measurements for the first hop, second hop, and direct links, respectively. Note that, compared to the simulated ray-based model given in Fig. 3, the experimental data has a significantly larger delay spread. Typical delay spreads of $80-100$ symbols were observed at a data rate of $1.2 \mathrm{~kb} / \mathrm{s}$. Furthermore, the $R-D$ channels are not perfectly synchronized, as can be seen in Fig. 12.

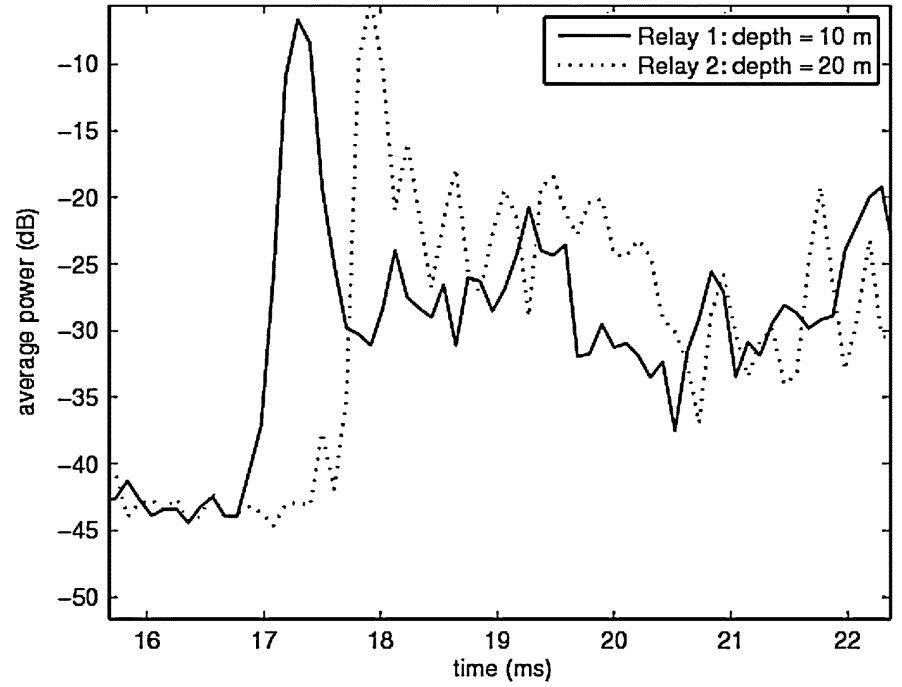

Fig. 12. Channel responses for asynchronous relays.

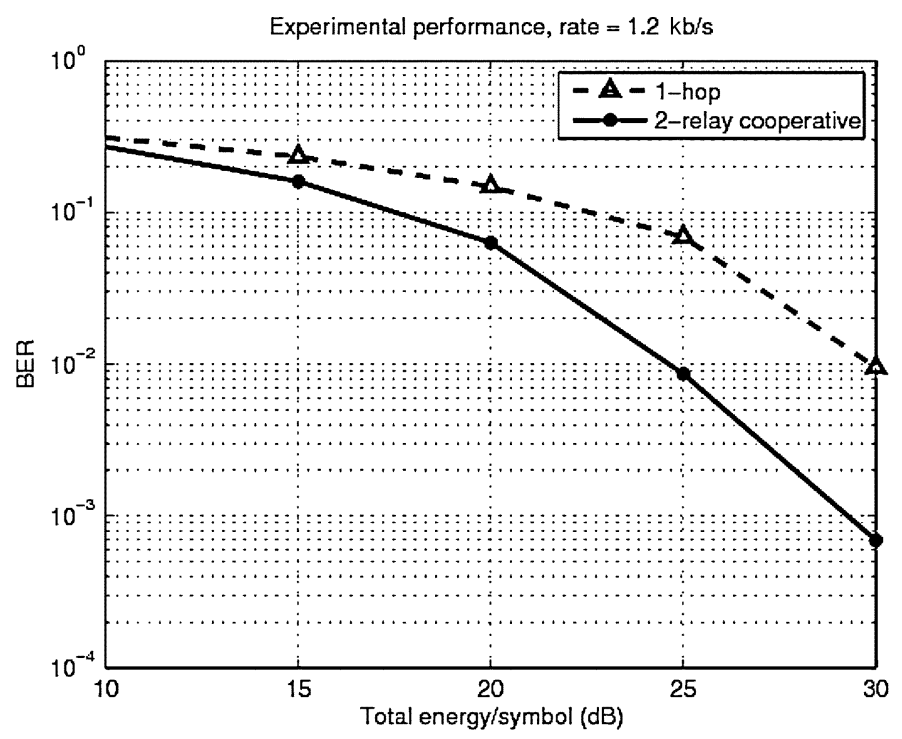

Fig. 13. Error rate performance of single-hop and cooperative transmission

In all cases, a strong main arrival is perceived, along with clusters of arrivals with delays of more than 100 ms. Furthermore, it can be observed that the direct $S-D$ channel was significantly weaker than the $S-R$ and $R-D$ channels. This constitutes a meaningful scenario for testing the multihop cooperation strategy.

The simulated performance with experimental channel data is shown in Fig. 13. The data rate is $1.2 \mathrm{~kb} / \mathrm{s}$ and the BPSK modulation scheme was employed. The data blocks length was set to $N=1000$ symbols, and a DFE with 50 forward taps was employed. Because perfect CSI was assumed, the number of feedback taps was set to be the delay spread (in symbols intervals) of the overall channel. Comparing the cooperative scheme performance with direct communication, both multihopping as well as diversity gains are observed. For the direct-hop case, a diversity (slope of the error probability curve) of around 1.2 
was observed, whereas it is around 2 for the cooperative case. The additional gain diversity observed in the direct-hop case can be fully attributed to multipath combining. For the cooperative strategy, in Section III, a diversity gain slightly smaller than 2 was predicted when no multipath gain was present. Clearly, therefore, the cooperative strategy is able to take advantage of diversity in both levels: space and the frequency selectivity of the channel.

\section{CONCLUSION}

In this paper, cooperative protocols for distributed space-time communications in underwater networks were proposed and analyzed. The proposed protocols, inspired by the time-reversal STBC [13], were shown to yield significant performance improvement over single-hop communication in underwater channels and can be generalized to networks with any number of relays. It was also shown, both numerically and experimentally, that the TR-DSTBC approach has the added advantage of not requiring a computationally expensive multidimensional equalization at the receiver, as well as being robust to asynchronism between the relays. Ongoing research focuses on the development of new, high rate, distributed protocols and space-time codes, which can be systematically applied to any number of relays.

\section{REFERENCES}

[1] I. F. Akyildiz, D. Pompili, and T. Melodia, "Underwater acoustic sensor networks: Research challenges," J. Ad Hoc Netw., vol. 3, no. 3, pp. 257-279, 2005

[2] E. M. Sozer, M. Stojanovic, and J. G. Proakis, "Underwater acoustic networks," IEEE J. Ocean. Eng., vol. 25, no. 1, pp. 72-83, Jan. 2000.

[3] H. Gharavi and K. Ban, "Multihop sensor network design for wideband communications," Proc. IEEE, vol. 91, no. 8, pp. 1221-1234, Aug. 2003.

[4] A. Sendonaris, E. Erkip, and B. Aazhang, "User cooperation diversity—Part 1: System description," IEEE Trans. Commun., vol. 51, no. 11, pp. 1927-1938, Nov. 2003.

[5] A. Sendonaris, E. Erkip, and B. Aazhang, "User cooperation diversity—Part 2: Implementation aspects and performance analysis," IEEE Trans. Commun., vol. 51, no. 11, pp. 1939-1948, Nov. 2003.

[6] J. Laneman and G. W. Wornell, "Distributed space-time coded protocols for exploiting cooperative diversity in wireless networks," IEEE Trans. Inf. Theory, vol. 49, no. 10, pp. 2415-2425, Oct. 2003.

[7] V. Tarokh, H. Jafarkhani, and A. R. Calderbank, "Space-time block codes from orthogonal designs," IEEE Trans. Inf. Theory, vol. 45, no. 5, pp. 1456-1467, Jun. 1999.

[8] Y. Hua, Y. Mei, and Y. Chang, "Wireless antennas: Making wireless communications perform like wireline communications," in Proc. IEEE Top. Conf. Wireless Commun. Technol., Honolulu, HI, Oct. 15-17, 2003, pp. 47-73.

[9] P. Anghel, G. Leus, and M. Kaveh, "Relay assisted uplink communication over frequency-selective channels," in Proc. IEEE Workshop Signal Process. Adv. Wireless Commun., Jun. 15-18, 2003, pp. 125-129.

[10] R. U. Nabar, H. Bölcskei, and F. W. Kneubuhler, "Fading relay channels: Performance limits and space-time signal design," IEEE J. Sel. Areas Commun., vol. 22, no. 6, pp. 1099-1109, Aug. 2004.

[11] Y. Jing and B. Hassibi, "Distributed space-time coding in wireless relay networks," IEEE Trans. Wireless Commun., vol. 5, no. 12, pp. 3524-3536, Dec. 2006.

[12] M. Vajapeyam and U. Mitra, "Performance analysis of distributed space-time coded protocols for wireless multi-hop communications," IEEE Trans. Wireless Commun., to be published.
[13] E. Lindskog and A. Paulraj, "A transmit diversity scheme for channels with intersymbol interference," in Proc. Int. Conf. Commun., Jun. 18-22, 2000, pp. 307-311.

[14] E. Larsson, P. Stoica, E. Lindskog, and J. Li, "Space-time block coding for frequency-selective channels," in Proc. Int. Conf. Acoust. Speech Signal Process., May 13-17, 2002, vol. 3, pp. 2405-2408.

[15] M. Stojanovic, "Retrofocusing techniques for high rate acoustic communications," J. Acoust. Soc. Amer., vol. 117, pp. 1173-1185, Mar. 2005.

[16] A. Zielinski, Y.-H. Yoon, and L. Wu, "Performance analysis of digital acoustic communication in a shallow water channel," IEEE J. Ocean. Eng., vol. 20, no. 4, pp. 293-299, Oct. 1995.

[17] S. M. Alamouti, "A simple transmit diversity technique for wireless communications," IEEE J. Sel. Areas Commun., vol. 16, no. 8, pp. 1451-1458, Oct. 1998.

[18] J. Proakis, Digital Communications. New York: McGraw-Hill, 1995.

[19] M. Stojanovic, L. Freitag, and M. Johnson, "Channel-estimation-based adaptive equalization of underwater acoustic signals," in Proc. OCEANS Conf., Sep. 1999, vol. 2, pp. 985-990.

[20] P. Monsen, "Theoretical and measured performance of a DFE modem on a fading multipath channel," IEEE Trans. Commun., vol. COM-25, no. 10, pp. 1144-1153, Oct. 1977.

[21] I. S. Gradshteyn and I. M. Ryzhik, Table of Integrals, Series and Products, 6th ed. New York: Academic, 2000.

[22] X. E. Li, "Space-time coded multi-transmission among distributed transmitters without perfect synchronization," IEEE Signal Process. Lett., vol. 11, pp. 948-951, Dec. 2004.

[23] Y. Mei, Y. Hua, A. Swami, and B. Daneshrad, "Combating synchronization errors in cooperative relays," in Proc. IEEE Int. Conf. Acoust. Speech Signal Process., Philadelphia, PA, pp. 369-372.

[24] M. Chitre, J. Potter, and O. S. Heng, "Underwater acoustic channel characterization for medium-range shallow water communications," in Proc. OCEANS Conf., Sep. 2004, vol. 1, pp. 40-45.

[25] T. H. Eggen, A. B. Baggeroer, and J. C. Preisig, "Communication over Doppler spread channels-Part I: Channel and receiver presentation," IEEE J. Ocean. Eng., vol. 25, no. 1, pp. 62-71, Jan. 2000.

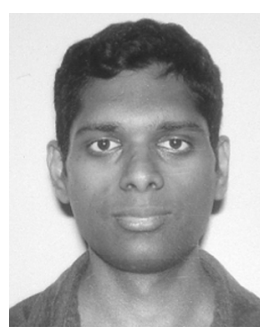

Madhavan Vajapeyam (S'99-M'06) received the B.S. degree from Universidade Federal da Paraiba (now Universidade Federal de Campina Grande), Brazil, in 2000 and the M.S. and Ph.D. degrees from the University of Southern California, Los Angeles, in 2002 and 2007, respectively, all in electrical engineering.

In summer 2005, he was an intern engineer with the Corporate R\&D Division, Qualcomm Inc., San Diego, CA, where he investigated multiantenna receiver techniques and spatial channel models for third-generation cellular systems. In 2007, he joined Qualcomm Inc., San Diego, CA, as a Senior Systems Engineer. He currently works on the design and standardization of next generation wireless orthogonal frequency-division multiple access (OFDMA) systems. His research interests include signal processing, cooperative communications, and design of physical and medium access protocols for wireless communications.

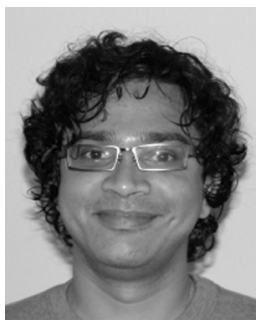

Satish Vedantam received the B.Tech. degree in electrical engineering from Indian Institute of Technology, Madras, India, in 2001 and the M.S. degree in electrical engineering and the M.S. degree in applied mathematics from the University of Southern California (USC), Los Angeles, both in 2003, where he is currently working towards the $\mathrm{Ph} . \mathrm{D}$. degree in electrical engineering.

In 2003-2005, he was a Research Analyst at Citigroup, New York, NY, where he worked with mortgage and interest rate derivatives. His research interests include information theory, estimation theory, and wireless communications. 
Urbashi Mitra (SM'04-F'07) received the B.S. and M.S. degrees from the University of California at Berkeley in 1987 and 1989 , respectively, and the Ph.D. degree from Princeton University, Princeton, NJ, in 1994.

From 1989 to 1990, she was a Member of Technical Staff with Bellcore, Red Bank, NJ. From 1994 to 2000, she was in the Electrical Engineering Faculty, Ohio State University, Columbus. In 2001, she joined the Department of Electrical Engineering, University of Southern California, Los Angeles, where she is currently a Professor. She has held visiting appointments at the Eurecom Institute, Rice University, and Stanford University.

Dr. Mitra was an Associate Editor for the IEEE TRANSACTIONS ON COMMUNICATIONS from 1996 to 2001; she is currently an Associate Editor for the IEEE JOURNAL OF OCEANIC ENGINEERING and the IEEE TRANSACTIONS ON INFORMATION THEORY. She served two terms as a Member of the IEEE Information Theory Society's Board of Governors. She is the recipient of: 2008 USC Mellon Mentoring Award, the Texas Instruments Visiting Professorship (Fall 2002, Rice University), 2001 Okawa Foundation Award, 2000 Lumley Award for Research (OSU), 1997 MacQuigg Award for Teaching (OSU), and 1996 National Science Foundation (NSF) CAREER Award. She cochaired the IEEE Communication Theory Symposium at the 2003 International Conference on Communications in Anchorage, AK, and cochaired the 1st Workshop on Underwater Networks at Mobicom 2006 in Los Angeles, CA. She is currently a Guest Editor for the upcoming IEEE JOURNAL OF SELECTED AREAS IN COMMUNICATIONS Special Issue on Underwater Networks and Communications.

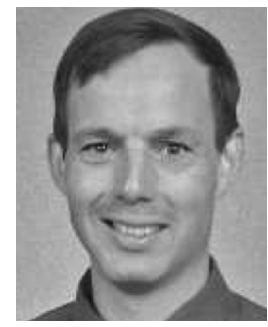

James C. Preisig (S'79-M'80) received the B.S degree in electrical engineering from the United States Coast Guard Academy, New London, CT, in 1980, the S.M. and E.E. degrees in electrical engineering from the Massachusetts Institute of Technology, Cambridge, in 1988, and the Ph.D. degree in electrical and ocean engineering from the Massachusetts Institute of Technology/Woods Hole Oceanographic Institution (WHOI) Joint Program in Oceanography and Oceanographic Engineering, Cambridge, MA, in 1992.

He was a Postdoctoral Investigator at WHOI from 1992 to 1994 and a Visiting Assistant Professor at Northeastern University, Boston, MA, from 1994 to 1997. Since July 1997, he has been on the scientific staff of the Department of Applied Ocean Physics and Engineering, WHOI, and is currently an Associate Scientist with Tenure. His research interests are in the areas of adaptive signal processing, system identification, underwater acoustic propagation modeling, underwater acoustic communications, and numerical optimization.

Dr. Preisig is the recipient of the 1999 U.S. Office of Naval Research Ocean Acoustics Young Faculty Award and is a member of the Acoustical Society of America's Underwater Acoustics and Signal Processing Technical Committees. $\mathrm{He}$ is also an Associate Editor of the IEEE JOURNAL OF OCEANIC ENGINEERINC and served as a member of the IEEE Sensor Array and Multichannel Signal Processing Technical Committee from 1998 to 2004.

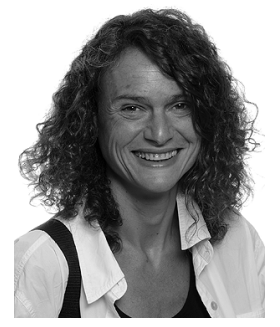

Milica Stojanovic (S'90-M'93-SM'08) graduated from the University of Belgrade, Belgrade, Serbia, in 1988, and received the M.S. and Ph.D. degrees in electrical engineering from Northeastern University, Boston, MA, in 1991 and 1993, respectively.

After a number of years with the Massachusetts Institute of Technology (MIT), Cambridge, where she was a Principal Scientist, in 2008, she joined the faculty of Electrical and Computer Engineering Department, Northeastern University. She is also a Guest Investigator at the Woods Hole Oceanographic Institution, Woods Hole, MA, and a Visiting Scientist at MIT. Her research interests include digital communications theory, statistical signal processing and wireless networks, and their applications to mobile radio and underwater acoustic communication systems.

Dr. Stojanovic is an Associate Editor of the IEEE JouRnal of OCEANIC ENGINEERING. 